\title{
Brand Addiction: A New Concept for Understanding Consumer Brand Behavior
}

\author{
Mona Mrad and Charles Cui
}

\begin{abstract}
This research introduces the concept of "brand addiction" as a new marketing construct. Employing conceptual development, the concept of brand addiction is defined as a psychological state that entails an obsessive relationship between the consumer and a specific brand. To examine the phenomenon of brand addiction, the present research was designed based on partial sequential mixed methods in which a qualitative study was followed by two quantitative studies, a pretest and a main survey. Arguing that research on brand addiction should be built based on an understanding of how consumers experience this phenomenon in reality, the research incorporated a series of four qualitative focus group studies to uncover the possible core features of the brand addiction prototype using discursive psychological perspective.

Survey data were subsequently used to generate the brand addiction measurement scale. Based on the qualitative study and critical review of the extant literature, we developed and tested a measurement scale of brand addiction to gauge the consumer's addictive behavior toward particular brands. As a result from exploratory and confirmatory factor analyses, the brand addiction measurement scale employed 14 items. The results appear to be promising for using this scale for further validation and more complicated research that may involve possible consequences caused by consumers' brand addictive behavior.

Therefore, rather than limiting to the interpersonal-relationship-based and addiction-based theories, this research has gone further in building an understanding of how consumers truly experience brand addiction, thus sheds new lights on this underrepresented phenomenon in the literature.
\end{abstract}

\footnotetext{
M. Mrad ( $\square)$

Lebanese American University, Beirut, Lebanon

e-mail: mona.mrad@lau.edu.lb

C. Cui

University of Manchester, Manchester, UK

e-mail: charles.cui@mbs.ac.uk

L. Petruzzellis, R.S. Winer (eds.), Rediscovering the Essentiality of Marketing, 